\title{
Adenine starvation is signalled through environmental stress response system in budding yeast Saccharomyces cerevisiae
}

\author{
Zane Ozolina, Agnese Kokina, Janis Liepins* \\ Institute of Microbiology and Biotechnology, University of Latvia, Jelgavas 1, Riga LV-1004, Latvia \\ ${ }^{\star}$ Corresponding author, E-mail: janis.liepins@lu.lv
}

\begin{abstract}
In the wild, budding yeast Saccharomyces cerevisiae often undergoes periods of nutrient abundance and absolute scarcity. It is capable of effectively halting its cell cycle in G1, in the case of lack of any basic nutrients. However, when lacking metabolic intermediates, the yeast behaves differently. Purine and not pyrimidine auxotrophic starvation in S. cerevisiae elicits rapid cell cycle arrest and increase of several stress (oxidative, acid, heat) resistances. Until now, molecular mechanisms governing formation of phenotype during auxotrophic adenine starvation in S. cerevisiae are not understood. The aim of the current research was to determine if the elements of environmental stress response system play a role during phenotype formation in adenine starvation in budding yeast. We tested if MSN2/4 or RIM15 C-end truncation affects desiccation tolerance in full media and after adenine starvation. We found that functional defects of each element of environmental stress response systems affected desiccation tolerance, however, C-end truncation of RIM15 lowered desiccation tolerance by several orders of magnitude, while MSN2/4 C-end truncation only by 2 to 4 times. Therefore, we hypothesize that there are other elements of the environmental stress response system except MSN4 and MSN2, responsible for adenine starvation specific, stress tolerant phenotype formulation.
\end{abstract}

Key words: adenine starvation, desiccation tolerance, MSN2, MSN4, RIM15, Saccharomyces cerevisiae.

Abbreviations: ESR, Environmental Stress Response; PKA, Protein Kinase A; SD, synthetic dextrose; RTG, Retrograde Signalling; TOR, Target of Rapamycin.

\section{Introduction}

Starvation is a typical event in the microbial lifetime, and it is estimated that most microorganisms on Earth are in the resting state initiated by lack of carbon/nitrogen/any other source necessary for biomass increase (Gray et al. 2004). In natural settings yeast often undergoes periods of nutrient abundance and absolute scarcity and is capable of effectively reacting to decrease of availability of carbon, sulphur and nitrogen sources. When starving for the aforementioned "basic" nutrients, yeast cells enter a stress resistant phenotype that aids survival till the external nutrient supply is restored (Boer et al.2010; Petti et al.2011). For genetic manipulation purposes various auxotrophies (interruptions of amino acid or nucleotide metabolism) have been introduced in yeast. Interestingly, when cells are starved for external supply of those auxotrophic metabolic intermediates (sometimes called "synthetic limitation"), for example, nucleotides or amino acids, yeast cells do not finish their cell cycle and stop division, and they continue to actively metabolize sugars and do not gain stress resistance. This phenotype has been described for uracil and leucine auxotrophic starvation and has earned the description "glucose wasting phenotype" (Boer et al. 2008).
Adenine auxotrophic starvation in many aspects induces a phenotype similar to cells in G0 arrest or quiescence. Saccharomyces cerevisiae cells with adenine auxotrophy (ade8 or ade2 mutants) when starving for adenine became desiccation tolerant, their budding index declines, and their chronological life span is increased (Kokina et al. 2014). The mechanism how and why cells translate lack of adenine into a desiccation and starvation resistant phenotype is yet unknown.

Various signaling pathways in the yeast cell link nutrient supply to cell growth rate and stress resistance. Target of Rapamycin, (TOR, nitrogen supply), Protein Kinase A (PKA, carbon supply), Retrograde Signalling (RTG) are the prominent examples of such pathways (see review by Broach 2012).

In the case of adenine starvation, we have observed more than a 200-fold increase in desiccation tolerance in S. cerevsiae cells (Kokina et al. 2014). Other authors have linked such a huge increase in desiccation tolerance to various genetic factors, including activity of targets of PKA and TOR pathways (Calahan et al. 2011; Welch et al. 2013). Msn2p, Msn4p and Rim15p are components of a system ensuring universal "Environment Stress Response" (ESR). The transcription factors Msn2p and Msn4p upregulate 
genes containing STRE sequences in their promoters (Berry, Gasch 2008). Rim15p is sensor-effector kinase that integrates nutrient and stress signals into widespread ESR response via activating endosulphines Igo1/2p. Those, in turn, provide stability for newly synthesised mRNA (Sarkar et al. 2014). Previously, Msn2/4p together with Rim15p, have been identified as crucial elements ensuring desiccation tolerance in S. cerevisiae (Welch et al. 2013).

Our aim was to test if ESR is involved in cell phenotype formation during adenine auxotrophic starvation. To test this, we determined if stable nuclear translocation of $\mathrm{Msn} 2 / 4 \mathrm{p}$ is necessary to ensure adenine starvation mediated desiccation tolerance. We constructed C-end truncations of MSN2/4 or RIM15, checked their identity and effect on growth. We found the cultivation time necessary for complete halt of cell proliferation (adenine starvation), thus giving us a timescale for adenine starvation specific effects. Finally we tested C-end truncation effects on desiccation tolerance of WT and each of newly constructed strains in fast growing and adenine starved cells.

\section{Materials and methods}

\section{Yeast strains and genetic constructions}

All experiments were carried out using $\Delta a d e 8$ S. cerevisiae strains of CEN.PK2 background (Kokina et al. 2014). Transcription factors were truncated by transforming yeast with linear PCR fragment containing flanking homologous sequences of the respective transcription factor and $6 \mathrm{xHis}$ tag and G418 marker in between (Janke et al. 2004). After transformation strain identity was confirmed by colony
PCR using gene specific test primers and internal primer (Nat_ctrl_Hb) from the insert (Lõoke et al. 2011). All the details regarding strains, plasmids and primers are given in the Table 1. All primers were synthesised by Sigma Aldrich.

\section{Growth conditions and media}

All starvation experiments were conducted in synthetic dextrose (SD) media (Verduyn et al. 1992) with all necessary auxotrophic agents added in surplus (Pronk 2002). For purine starvation, synthetic media without adenine was used.

Growth rate analyses were performed in a 96 well multimode reader TECAN Infinite M200 PRO. S. cerevisiae cells were grown in synthetic media containing glucose, washed three times with distilled water and resuspended in the synthetic media with all combinations of amonia/ proline and glucose/galactose as $\mathrm{N}$ and $\mathrm{C}$ sources.

To assess adenine starvation effect on desiccation tolerance, cells were grown in full SD media up to exponential phase $\left(\mathrm{OD}_{600}\right.$ less than 1$)$, washed with distilled water, and re-suspended to $\mathrm{OD}_{600}=0.5$ in SD media without adenine, but with all other broth components added. The yeasts were incubated for $4 \mathrm{~h}$ in a rotary shaker $(140 \mathrm{rpm}$, $30^{\circ} \mathrm{C}$ ) and then desiccated.

\section{Desiccation tolerance assay}

Desiccation tolerance was assayed by estimating $\mathrm{CFU} \mathrm{mL} \mathrm{mL}^{-1}$, before desiccation and after dehydration. One millilitre of culture at $\mathrm{OD}_{600}=1$ was washed with distilled water twice, diluted serially and spotted on YPD (yeast extract $1 \%$, bactopeptone $2 \%$, glucose $2 \%$, agar $2 \%$ ) plates. The

Table 1. Yeast strain, plasmids and primers used in the present study

\begin{tabular}{|c|c|c|c|}
\hline & Name & Description/sequence/ & Source \\
\hline Yeast strain & WT & 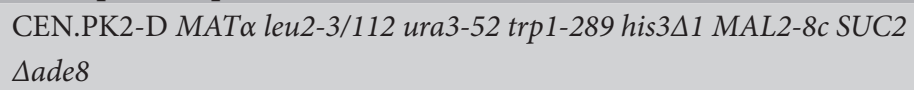 & Kokina et al. 2014 \\
\hline Plasmid & pYM46 & $\begin{array}{l}\text { PCR template for C-terminal myc tag plus } 7 \text { His residues: marker } \\
\text { pAgTEF-kanMX-tAgTEF, selectable phenotype: G418 resistance }\end{array}$ & $\begin{array}{l}\text { Janke et al. 2004, } \\
\text { EUROSCARF }\end{array}$ \\
\hline \multirow[t]{10}{*}{ Primers } & MSN4_S2 & $\begin{array}{l}\text { CTTGTCTTGCTTTTATTTGCTTTTGACCTTATTTT } \\
\text { TTTCAATCGATGAATTCGAGCTCG }\end{array}$ & This study \\
\hline & MSN4_S3 & $\begin{array}{l}\text { GCATTCAGACGCAGTGAGCACTTGAAAAGGCATA } \\
\text { TAAGATCGTACGCTGCAGGTCGA }\end{array}$ & This study \\
\hline & MSN2_S3 & $\begin{array}{l}\text { GAAATTTAGTAGAAGCGATAATTTGTCGCAACAC } \\
\text { ATCAAGCGTACGCTGCAGGTCGA }\end{array}$ & This study \\
\hline & MSN2_S2 & $\begin{array}{l}\text { TGAAGAAAGATCTATCGAATTAAAAAAATGGGGT } \\
\text { CTATTAATCGATGAATTCGAGCT }\end{array}$ & This study \\
\hline & RIM15_S2 & $\begin{array}{l}\text { CAGTTATTTTTTTTAATTATCTTTATCTTAAAATT } \\
\text { TATCAATCGATGAATTCGAGTCCG }\end{array}$ & This study \\
\hline & RIM15_S3 & $\begin{array}{l}\text { CAGGAGGCGGCAACCAGTAGAGTCTTTGACGAT } \\
\text { GTTTTAGCGTACGTCGCAGGTCGA }\end{array}$ & This study \\
\hline & MSN4_test_F01 & AGAAGGCATTCAGACGCAGT & This study \\
\hline & RIM15_test_F01 & CCAATTGTGGCCATAACAAA & This study \\
\hline & Jli_MSN2-t01 & CCATTATCGCCTGCATCATCAT & This study \\
\hline & NAT-HgB_ctrl & ACGAGGCAAGCTAAACAGATCT & This study \\
\hline
\end{tabular}


remaining cell suspension was centrifugated, and the pellet was left to desiccate for $6 \mathrm{~h}$ at $30^{\circ} \mathrm{C}$ in a desiccator, then diluted in distilled water in room temperature. Rehydrated cell solution was thoroughly mixed, serially diluted and spotted on YPD plates. The viability (\%) was calculated by dividing the number of $\mathrm{CFU} \mathrm{mL}^{-1}$ after and before desiccation, similarly to Calahan et al. (2011).

\section{Statistics}

All experiments were performed in triplicates, error bars represent standard deviations. Asterix is used where $p<$ 0.05 .

\section{Results and discussion}

\section{Adenine starvation halts cell proliferation}

At first we determined effect of adenine starvation on the wildtype cells growth dynamics. Results are shown in the Fig. 1.

S. cerevisiae wildtype strain used in this study has multiple auxotrophies. It is auxotroph for histidine, adenine, tryptophan, leucine and uracil. As a well characterised control for yeast cell auxotrophic starvation, we added uracil deficient media and observed the growth pattern in this medium. When cultivated in adenine or uracil deficient media, auxotrophic starvation led to halt of cell proliferation, as cells lack building blocks for further growth. Previously we have observed that optical density alone is not a reliable measurement of actual cell number in the case of adenine-starved cells. While starving for adenine, $S$. cerevisiae cells tend to increase in volume and therefore cell number per $\mathrm{mL}$ in unit of absorbance

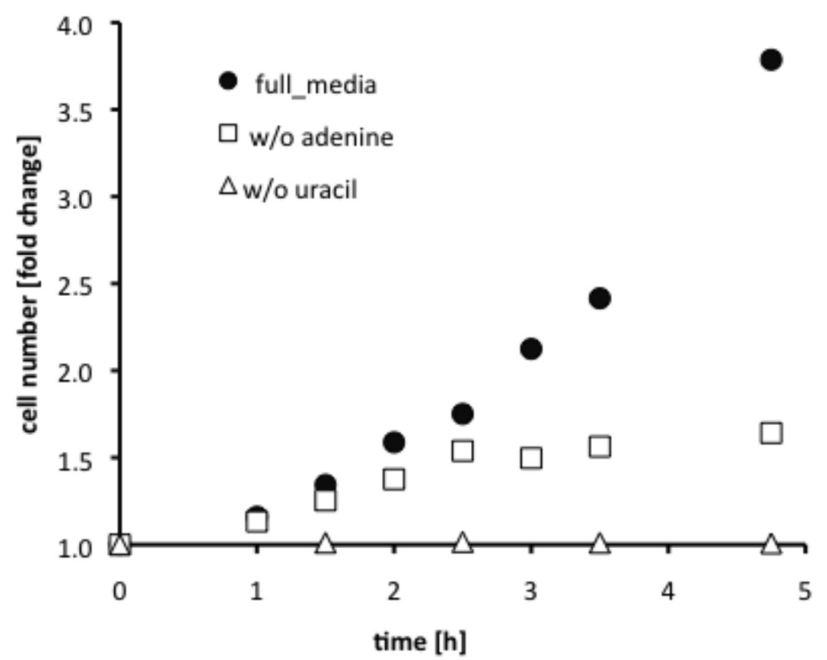

Fig. 1. S. cerevisiae wildtype growth dynamics in full SD media and in the SD media without adenine or uracil supplement (w/o adenine or w/o uracil). Growth dynamics were registered with a $\mathrm{Z}^{\mathrm{mi}}$ Coulter counter ${ }^{\circledast}$ (Beckman Coulter). It is depicted as fold change (cell number of the each time point normalised to initial cell number). Data points are average from independent triplicates; standard deviation is less than $4 \%$, error bars not visible. value is affected (Kokina et al. 2014). To be sure that we observed only halt of cell proliferation rather than increase in cell volume, we used direct cell counts via particle counter, not spectrophotometrical OD measurements. To compare cultivations with different initial cell numbers, we normalised all cell number per $\mathrm{mL}$ against initial cell number (see Fig. 1).

Cells cultivated in adenine free, synthetic dextrose media cease to multiply later than in the case of uracil starvation. Based on our data (Fig.1), we assumd that adenine starvation sets in 2 to $3 \mathrm{~h}$ after a shift to starvation media, while in the case of uracil starvation, it set in immediately after media shift. Therefore, to induce a "adenine starvation phenotype", we cultivated wildtype cells in adenine deficient media for $4 \mathrm{~h}$.

\section{Msn2/4p and Rim15p C-end truncation affects cell growth}

Transcription factors were truncated by transforming yeast with the linear PCR fragment containing flanking homologous sequences of the respective transcription factor and 6xHis tag and G418 marker in between (Janke et al. 2004). When inserting fragments, the last $100 \mathrm{bp}$ of coding sequence were removed. The last 50 amino acids of MSN2/4p code for DNA binding Zn finger domain and are responsible for binding to target STRE sequences (Estruch, Carlson 1993). Rim15p contains REC domain in it's C-end; it seems to be involved in autophosphorylation and dimerisation activities (Wanke et al. 2005).

We tested growth of truncated strains in synthetic media with varying carbon and nitrogen sources. We chose glucose and galactose as repressive and derepressive examples of carbon sources and ammonium sulphate as example of repressive and proline as non-repressive nitrogen source (Gancedo 1998; Cooper 2002). As a result, we observed different growth patterns when strains where grown under different $\mathrm{C}$ or $\mathrm{N}$ sources. This reflects divergent functions that these factors have in adopting cell growth in the presence of different (repressive or derepressive) carbon or nitrogen sources, see Fig. 2. Previously we had confirmed identity of our constructs by colony PCR (Lõoke et. al. 2011); these media shift studies served as functional confirmation of successful gene truncation and effect on the cell signalling system.

In synthetic media with glucose and ammonia as carbon and nitrogen sources, the Msn2p C-end truncated strain exhibited similar specific growth rate $(\mu)$ as the wildtype. Strains with Msn4p, and Rim15p C-end truncated grew $25 \%$ slower (see Fig. 2), $0.4 \mathrm{~h}^{-1}$ for the wildtype and $m s n 2$, $0.28 \mathrm{~h}^{-1}$ and $0.26 \mathrm{~h}^{-1}$ for $m s n 4$ and rim15, respectively. Similar effects of Msn2 functional impairments on strain growth have been demonstrated previously by Estruch and Carlson (1993). In both cases, C-end specific truncation (our results) and msn2/4 partial knockouts (Estruch, Carlson 1993) resulted in hampered growth of the strains on galactose as the main carbon source.

In all carbon-nitrogen source combinations, the 


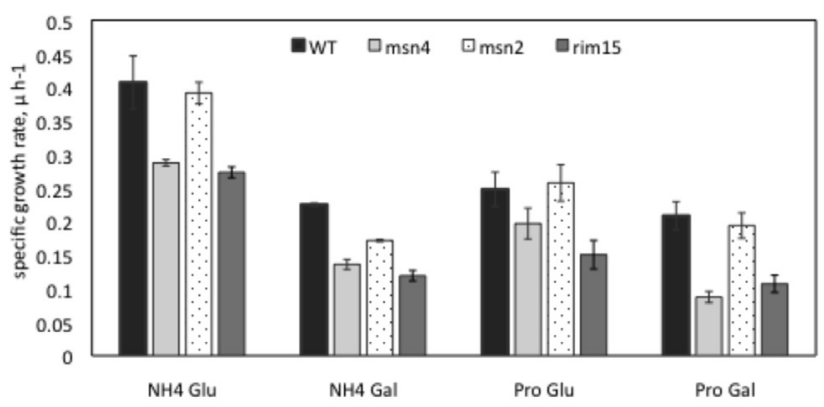

Fig. 2. Growth rate $\left(\mu \mathrm{h}^{-1}\right)$ of the wildtype (WT) strain and it's corresponding strains with C-end truncated $m s n 2, m s n 4$, rim 15 mutants cultivated in SD+ media with different carbon (Glu, glucose; Gal, galactose) and nitrogen $\left(\mathrm{NH}_{4}\right.$, ammonium; Pro, proline) sources. Each bar depicts the mean of four independent cultivations. Error bars represent standard errors.

Rim15p C-end truncated strain grew slower and exhibited a comparatively long lag phase. Interestingly, low growth speed and extended lag phase have been observed with sake yeast possessing a frame shift mutation in the Rim 15p $\mathrm{C}$-end and lacking 75 amino acids of the C-end (Watanabe et al. 2012).

The nitrogen source shift affected Msn2/4p C-end truncated strains in the same way as the wildtype. Derepressing the nitrogen source together with derepressing the carbon source decreased growth of all strains, since carbon and nitrogen metaboslims share common metabolites, like a ketoglutarate (Cox et al. 2002; Tate, Cooper 2013). The results showed that the introduced changes are more responsive to changes in the carbon, not nitrogen source.

\section{Msn2/4 and Rim $15 \mathrm{C}$-end truncation affects S. cerevisiae cell desiccation tolerance after adenine starvation}

Desiccation tolerance is a typical feature of S. cerevisiae, which is often used for biotechnological purposes (like preparation of dried yeast for bread, beer and wine production). To survive desiccation, cells should be ready for a number of stresses (oxidative, salt, heat, etc.; review in Dupont et al. 2014). It is also known that condition of a cell before desiccation directly correlates to survival of desiccation tolerance. (Welsh et al. 2013). Here we use desiccation "stress" as a "environmental stress" to determine the activity state of ESR.

We attempted to determine if transcription factors Msn2/4p or their upstream kinase Rim15p have any role in adenine starvation induced desiccation tolerance in $S$. cerevisiae. We grew wildtype and derived strains with C-end truncated Msn2/4p or Rim15p till $\mathrm{OD}_{600}$ approx 1 or less, harvested the cells, washed them, and resuspended them in SD complete broth or SD lacking adenine with final $\mathrm{OD}_{600}=0.5$. After cultivation in shake flasks on rotary shaker for $4 \mathrm{~h}, 30^{\circ} \mathrm{C}$, we harvested cells from all the shake flasks, desiccated and spotted them on solid YPD agar and counted colonies. Viability was calculated as proportion of culture CFU after desiccation against CFU before desiccation. Desiccation tolerance results are shown in Fig. 3.

After adenine starvation, we obtained similar desiccation tolerance results for wildtype as published previously (Kokina et al. 2014). We observed a huge (more than 1000 times) increase in desiccation tolerance in the adenine starved cells when compared to desiccation tolerance of rapidly growing wildtype cells.

Desiccation tolerance of all C-end truncated strains were statistically significantly different from the wildtype. In the case of strains with C-end truncated Msn2/4p, desiccation tolerance after adenine starvation was lower than in wildtype cells. Desiccation is rather complex treatment, which includes elements of oxidative, heat shock and osmotic stresses (Franca et al. 2005). Therefore, functional impairment of transcription factors responding to all those stresses (Msn2p and Msn4p) will inevitably lead to lowered tolerance. However, drop of the viability in these strains was within the same order of magnitude. Since our spot test allowed us to estimate population viability from $100 \%$ down to $0.00001 \%$, therefore a "significant signal" when using such a rough metric indicated changes in orders of magnitudes and not changes within the same order of magnitude. Calahan et al. (2011) observed a drop in desiccation tolerance in $m s n 2$ and $m s n 4$ knockouts within the same order of magnitude in saturated cultures, similarly to our C-end MSN2/4 truncations.

Interestingly, Rim15p C-end truncation led to increase in desiccation tolerance in exponentially growing culture (SD media with all necessary supplements). It reached $0.03 \%$, which is approximately 50 times more than typical desiccation tolerance of the wildtype and other strains when grown in full media. In comparison, the Rim15p

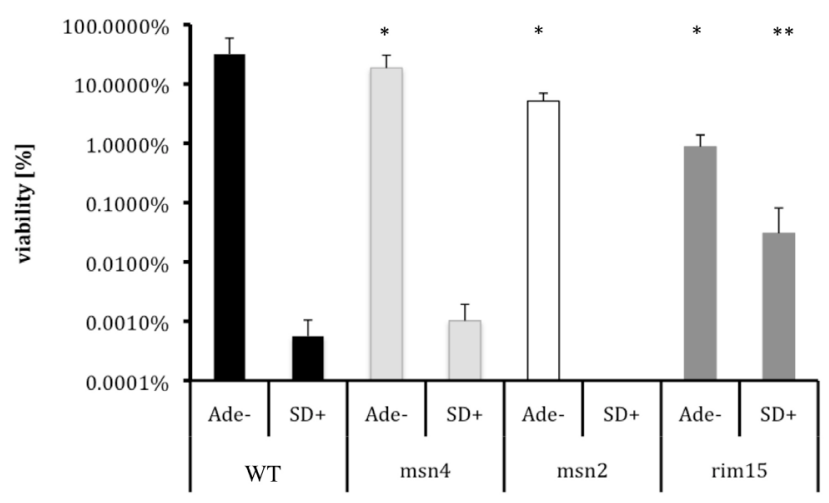

Fig. 3. Desiccation tolerance of wikdtype (WT) and it's derivative strains with C-end truncated msn2, msn4 or rim15, after $4 \mathrm{~h}$ cultivation in full SD media (SD+) or SD media without adenine (Ade-). Desiccation was performed at $30^{\circ} \mathrm{C}$ in a desiccator. Each bar represents the mean of three independent samples, error bars represent standard deviations. Stars depict significant differences $(p<0.05)$ between wildtype and engineered strain adenine starvation treatment $\left(^{*}\right)$ and full media $\left(^{* *}\right)$. 
C-end truncated strain desiccation tolerance after adenine starvation increased just 30, not 1000 or more times, when compared to wildtype.

Truncation of Rim $15 \mathrm{p}$ led to a more severe desiccation tolerance decline than in the $m s n 2 / 4$ double knockout (Calahan, et al. 2011). This indicates that there are other elements of Environmental Stress Response system responsible for adenine starvation specific, stress tolerant phenotype formulation, except besides MSN4 and MSN2.

Sake yeasts form a compact $S$. cerevisiae strain subgroup, where at least seven strains have C-end truncated versions of Rim15p (Liti et al. 2009; Wang 2012; Watanabe 2012). We observed that the strain with Rim 15 p C-end truncation had increased desiccation tolerance in it's exponential phase. While several sake yeast strains ferment glucose to ethanol rapidly, they are characterised for low ethanol and heat stress tolerance. This has been linked to defects in Msn2/4p signalling (Watanabe 2011). Also, these strains enter quiescent state with low efficiency (Urbanzcyk, 2011). Recently, genetic defects in the Msn2/Msn4 upstream regulator RIM15 have been found. When transforming cells with the "correct" version of RIM15, growth, heat and ethanol tolerance defects were complemented (Watanabe 2012).

Our results allow us to speculate that the Rim15p C-end truncated version might have phenotypic advantage during sake production. We presume that improved ethanol production together with comparatively "increased" desiccation tolerance in the exponential phase could be a phenotype being unintentionally selected throughout the rich history of sake production (Kitigaki, Kitogoto 2013).

\section{Acknowledgements}

We are very thankful to Prof. Arnold Kristjuhan (Institute of Molecular and Cell biology, University of Tartu) for access to a cell counter. Zane Ozolina was supported by Laiminu family scholarship and scientific grant from the Student Council of University of Latvia. Agnese Kokina was supported by the Latvian basic science grant No. ZD2016AZ03 and research performance grant No. AAP2016/B041. Janis Liepins was supported by a FP7 project InnovaBalt, Latvian National Research Programme (BIOMEDICINE 2014-2017) and \#PostDocLatvia funding scheme (ERDAF proj. No. 1.1.1.2/16/1/001, subproject 1.1.2/1/16/067 \#YEAUX). .

\section{References}

Berry D.B., Gasch A.P. 2008. Stress-activated genomic expression changes serve a preparative role for impending stress in yeast. Mol. Biol. Cell 19: 4580-4587.

Boer V.M., Amini S., Botstein D. 2008. Influence of genotype and nutrition on survival and metabolism of starving yeast. Proc. Nat. Acad. Sci. USA 105: 6930-6935.

Boer V.M., Crutchfield C.A., Bradley P.H., Botstein D., Rabinowitz J.D. 2010. Growth-limiting intracellular metabolites in yeast growing under diverse nutrient limitations. Mol. Biol. Cell 21: 198-211.
Broach J.R. 2012. Nutritional control of growth and development in yeast. Genetics 192: 73-105.

Calahan D., Dunham M., De Sevo C., Koshland D.E. 2011. Genetic analysis of desiccation tolerance in Sachharomyces cerevisiae. Genetics 189: 507-519.

Cox K.H., Tate J.J., Cooper T.G. 2002. Cytoplasmic compartmentation of Gln3 during nitrogen catabolite repression and the mechanism of its nuclear localization during carbon starvation in Saccharomyces cerevisiae.J. Biol. Chem. 277: 37559-37566.

De Virgilio C. 2011. The essence of yeast quiescence. FEMS Microbiol. Rev. 36: 306-339.

Dupont S., Rapoport A., Gervais P., Beney L. 2014. Survival kit of Saccharomyces cerevisiae for anhydrobiosis. Appl. Microbiol. Biotechnol. 98: 8821-8834.

Estruch F., Carlson M. 1993. Two homologous zinc finger genes identified by multicopy suppression in a SNF1 protein kinase mutant of Saccharomyces cerevisiae. Mol. Cell Biol. 13: 38723881.

Franca M.B., Panek A.D., Eleutherio E.C.A. 2005. The role of cytoplasmic catalase in dehydration tolerance of Saccharomyces cerevisiae. Cell Stress Chaper. 10: 167-170.

Gancedo J.M., 1998. Yeast carbon catabolite repression. Microbiol. Mol. Biol. Rev. 62: 334-361.

Gray J.V., Petsko G.A., Johnston G.C., Ringe D., Singer R.A., Werner-Washburne M. 2004. "Sleeping beauty": quiescence in Saccharomyces cerevisiae. Microbiol. Mol. Biol. Rev. 68: 187206.

Janke C., Magiera M.M., Rathfelder N., Taxis C., Reber S., Maekawa H., Knop M. 2004. A versatile toolbox for PCR-based tagging of yeast genes: New fluorescent proteins, more markers and promoter substitution cassettes. Yeast 21: 947-962.

Kitagaki H., Kitamoto K. 2013. Breeding research on sake yeasts in Japan: history, recent technological advances, and future perspectives. Annu. Rev. Food Sci. Technol. 4: 215-235.

Kokina A., Kibilds J., Liepins J. 2014. Adenine auxotrophy - be aware: some effects of adenine auxotrophy in Saccharomyces cerevisiae strain W303-1A. FEMS Yeast Res. 14: 1-11.

Liti G., Carter D.M., Moses A.M., Warringer J., Parts L., James S.A., Davey R.P., Roberts I.N., Burt A., Koufopanou V., Tsai I.J. 2009. Population genomics of domestic and wild yeasts. Nature 458: 337-341.

Lõoke M., Kristjuhan K., Kristjuhan A. 2011. Extraction of genomic DNA from yeasts for PCR-based applications. Biotechniques 50: 325-328.

Petti A.A., Crutchfield C.A., Rabinowitz J.D., Botstein D. 2011. Survival of starving yeast is correlated with oxidative stress response and nonrespiratory mitochondrial function. Proc. Nat. Acad. Sci. USA 108: 1089-1098.

Pronk J.T. 2002. Auxotrophic yeast strains in fundamental and applied research. Appl. Environ. Microbiol. 68: 2095-2100.

Sarkar S., Dalgaard J.Z., Millar J.B.A., Arumugam P. 2014. The rim15-endosulfine-PP2ACdc55 signalling module regulates entry into gametogenesis and quiescence via distinct mechanisms in budding yeast. PLoS Genet. 10: e1004456.

Tate J.J., Cooper T.G. 2013. Five conditions commonly used to down-regulate tor complex 1 generate different physiological situations exhibiting distinct requirements and outcomes. J. Biol. Chem. 288: 27243-27262.

Urbanczyk H., Noguchi C., Wu H., Watanabe D., Akao T., Takagi H., Shimoi H. 2011. Sake yeast strains have difficulty in entering a quiescent state after cell growth cessation. J. Biosci. 
Bioeng. 112: 44-48.

Verduyn C., Postma E., Scheffers W.A., Van Dijken J.P. 1992. Effect of benzoic acid on metabolic fluxes in yeasts: A continuousculture study on the regulation of respiration and alcoholic fermentation. Yeast 8: 501-517.

Wanke V., Pedruzzi I., Cameroni E., Dubouloz F., De Virgilio C., 2005. Regulation of G0 entry by the Pho80-Pho85 cyclinCDK complex. EMBO J. 24: 4271-4278.
Watanabe D., Araki Y., Zhou Y., Maeya N., Akao T., Shimoi H. 2012. A loss-of-function mutation in the PAS kinase Rim15p is related to defective quiescence entry and high fermentation rates of Saccharomyces cerevisiae sake yeast strains. Appl. Environ. Microbiol. 78: 4008-4016.

Welch A.Z., Gibney P.A., Botstein D., Koshland D.E. 2013. TOR and RAS pathways regulate desiccation tolerance in Saccharomyces cerevisiae. Mol. Biol. Cell 24: 115-28. 